\title{
Primary mechanism of action of optical radiation on living organisms
}

\begin{abstract}
In today's technological development of human society more and more influence on the lives of biological organisms different electromagnetic radiation. Therefore, the study and analysis of the mechanisms of their effects is an urgent task.
\end{abstract}

Volume 4 Issue 4 - 2018

\section{Chervinsky LS}

National University of Life and Environmental Sciences of Ukraine, Ukraine

Correspondence: Chervinsky LS, National University of Life and Environmental Sciences of Ukraine, Ukraine, Email Ichervinsky@gmail.com

Received: April 20, 2018 | Published: August 08, 2018

\section{Introduction}

The study of the primary mechanisms of interaction of photons of optical radiation with the structures of biological objects, using the laws of quantum mechanics and biophysics.

\section{Materials and methods}

Studies are presented on the basis of the use of the provisions of the system analysis.

\section{Results}

Interaction of electromagnetic radiation (EMR) from the optical range of biologic objects as shown in the wave, and quantum effects in the probability of formation of which varies depending on the wavelength. In assessing the effectiveness of EMR in the optical range, along with such laws of its wave propagation, as a reflection, scattering and absorption, it is also necessary to take into account the effects of corpuscular - photochemical, photoelectric, photolytic and others. Photobiological basis of the mechanism of action of EMR optical range is the energy absorption of light quanta (photons) by atoms and molecules of biological structures (law Grotgus-Draper), which resulted in the formation of electronically excited states of these molecules with the transfer of photon energy (internal photo effect). This is accompanied by electrolytic dissociation and ionization of biological molecules. The degree of manifestation of photo biological effects in the body depends on the intensity of the optical radiation, which is inversely proportional to the square of the distance from the source to the irradiated surface. Accordingly, in practice, determine not the intensity and irradiation dose at a certain distance from the source of exposure by the exposure time.

As a result, we can say that the interaction of EMR optical range with biological tissues of living organisms transformed into other forms of energy (mechanical, chemical, thermal, and others.). Processes caused by the excitation of molecular structures or heating of body tissues, are the starting link physico-chemical and biological reactions, which form the final therapeutic effect. In addition, each of the types of optical radiation is considered to be unique to him physical and chemical processes that determine the specificity of their therapeutic effects and practical application. The phenomenon of selective absorption of optical radiation energy different types of sensing cells and biological structures suggests different mechanisms of their impact and formed their therapeutic effects. This effect is a combination of developing the radiation interrelated processes. The probability of their formation and development is determined by the peculiarities of the energy distribution of the optical radiation in space and time, physical (electrical, magnetic, mechanical, thermal) properties of biological tissues - "targets", selective sensitivity to him and functional reserves of adaptation and reactivity. In the formation of therapeutic effects from exposure to optical radiation energy are making a significant contribution to the processes of various levels of organization of a living organism, starting with the primary biophysical and ending with complex adaptation. ${ }^{1-3}$

\section{Acknowledgements}

The author would like to acknowledge Federal University of ABC (UFABC) for provided facilities and equipments.

\section{Conflict of interest}

Author declares that there is no conflict of interest.

\section{References}

1. Chervinsky LS. The action lights on the derma animal's. Extra on international conf. 1st Congress of the World Association for Laser Therapy «WALT»; 1996 May 5-9, Jerusalem, Israel. 1996. p. 2922-3002.

2. Chervinsky LS. Investigation of the Light-Conductivity of the Separate Hair and Skins Translucence. PITTCON'98, 1998 March 1-5; New Orleans, Louisiana, USA. p. 652.

3. Chervinsky LS. About the mechanism of photoreactivation of the biological objects. Mother international conf." The European Biomedical Optics Week, BIOS Europe'97', 1997 September 4-8; San Remo, Italy. 1997. p. 3198-3030. 